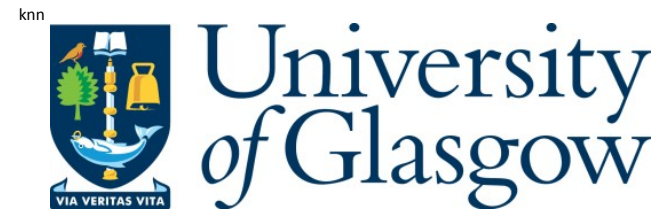

Hu, R., K ouzapas, D., Pernet, O., Y oshida, N ., and Honda, K. (2010) TypeSafe Eventful Sessions in J ava. In: ECOOP 2010 - Object-Oriented Programming, 24th European Conference, 21-25 J une 2010, M aribor, Slovenia.

Copyright ( 2010 Springer V erlag

http://eprints.gla.ac.uk/101380/

Deposited on: 22 J anuary 2015

Enlighten - Research publications by members of the University of Glasgow http://eprints.gla.ac.uk 


\title{
Type-Safe Eventful Sessions in Java
}

\author{
Raymond Hu*, Dimitrios Kouzapas*, Olivier Pernet* \\ Nobuko Yoshida*, and Kohei Honda ${ }^{\dagger}$ \\ *Imperial College London $\quad \nmid$ Queen Mary, University of London
}

\begin{abstract}
Event-driven programming is a major paradigm in concurrent and communication-based programming, and a widely adopted approach to building scalable high-concurrency servers. However, traditional event-driven programs are more difficult to read, write and verify than their multi-threaded counterparts due to low-level APIs and fragmentation of control flow across disjoint event handlers. This paper presents a Java language extension and a novel type discipline for type-safe event-driven session programming that counters the problems of traditional event-based programming with abstractions and safety guarantees based on session types, while retaining the expressiveness and performance characteristics of events. The type discipline extends session types and their primitives with asynchronous input, session typecase and session set types, ensuring eventhandling safety and event progress in addition to the standard type soundness and communication safety. The advantages, expressiveness and performance of event-driven session programming are demonstrated through a range of examples and benchmarks, including a session-typed SMTP server.
\end{abstract}

\section{Introduction}

Asynchronous event-driven programming is characterised by a reactive flow of control driven by the occurrence of computation events. It is one of the major paradigms in concurrent and communication-based programming, where events are typically detected by the arrival of messages on asynchronous channels. Primary motivations for asynchronous event programming include performance and scalability, particularly for high-concurrency applications such as Web servers [19,31]. Unfortunately, the flexibility and performance of traditional event-driven programming comes at the cost of more complex programs: low-level APIs and the obfuscation of event-driven control flow [2,29] make programs difficult to read, write and verify, and hence potentially unsafe to execute. Consequently, several recent works [20, 22, 30] have proposed simpler thread-based programming interfaces that hide event-driven runtimes. In contrast to these approaches, our aim in this paper is to develop a high-level, structured and safe programming discipline for event-driven programming based on, and extending, session types $[14,28]$. We generalise the existing session types for asynchronous event-driven programming, using which we obtain both formal safety guarantees and programmatic benefits to overcome the problems of traditional event-driven programming.

Session types $[14,28]$ are one of the well-studied type-based methods for structuring a series of distributed interactions. Previous works have studied the theory and practice of session types in object-oriented languages $[7,9,11,16]$ that ensure the so-called communication safety, meaning that communicating programs correctly interact following 
the associated session type structures. However, typing asynchronous event-driven programming is not possible so far, for the reasons outlined below.

A general mechanism underlying all event-based systems is the asynchronous detection of heterogeneous events (i.e. of varied types) from a dynamic collection of channels. This idea is embodied by e.g. the Unix select system call and the Java NIO Selector API. In the context of session programming, this means we need a framework where we collect multiple channels of different session types, asynchronously check for the arrival of messages on these channels, and later retrieve and use the "ready" channels as directed by their session types. The preceding session type disciplines cannot support these ideas because the lack of non-blocking input prohibits core event idioms such as event loops, and because statically determined channel types make it impossible to treat a collection of channels with heterogeneous types.

Contributions. This paper develops a framework for type-safe event-driven session programming that integrates session types and asynchronous event programming in Java. The key concepts of event-driven session programming are introduced through initial motivating examples in $\S \mathbf{2}$. The rest of the paper presents the following contributions.

(§3) We explore a theoretical basis of event-driven session programming using a small process calculus based on $[13,14,28]$. The formalism captures the semantics of asynchronous, event-driven sessions through two new constructs, the message arrival predicate and session typecase. Combining the latter with the new session set types allows us to treat dynamic collections of heterogeneously typed channels.

(§4) A type theory based on session set types for the extended session constructs. In addition to type soundness and communication safety in the presence of dynamically registered sessions, we prove a novel progress property which we call event progress. The theory captures a wide range of event-driven programming idioms (e.g event selectors, event loops and join patterns) via encoding, from which we can derive sound typing rules for such complex constructs.

(§5) Building on the new theory, we present the design and implementation of a practical language, compiler and runtime support for event-driven session programming as an extension to Java, on the basis of SJ (Session Java)[16]. The resulting Eventful $S J$ (ESJ) enjoys the formal properties and safety guarantees established by the theory. We discuss an ESJ implementation of an SMTP server and show that ESJ preserves the performance characteristics of traditional event-driven programming by benchmarking the ESJ server against a multithreaded equivalent.

Our implementation of ESJ exploits the modular architecture of the SJ runtime [25] for transport independence: the ESJ selector enables the event-driven execution of sessions not only of different types but also over different transports, under a single programming abstraction (§5.2). Implementing ESJ reveals further applications of the eventful session theory. For example, runtime session type monitoring enables applications like the SMTP server to execute sessions with non-SJ parties while protecting communication safety $(\S 5.3)$. Related work and future topics are discussed in $\S 6$. Omitted definitions, proofs, ESJ source code and benchmark results can be found at [25]. 


\section{Event-driven Session Programming}

This section introduces the key concepts of event-driven session programming through examples, presented in the syntax of Eventful SJ (ESJ for short), illustrating both the safety guarantees and practical programming benefits of our new event-driven programming framework compared to the traditional one.

ESJ is built on SJ, an extension of Java for type-safe concurrent and distributed session programming [16]. Session programming in SJ, as detailed in [16, § 2], starts with the declaration of the intended communication protocols as session types. The communication actions comprising a session, such as message passing, branching and recursion, are implemented as operations on session-typed channel endpoints called session sockets, objects of type SJSocket. The SJ compiler statically checks session implementations against the declared protocols, ensuring correct communication behaviour. ESJ extends SJ with facilities for session-typed asynchronous event handling.

Event loops. The core of any event-based system is the event loop [21], which waits for event occurrences (i.e. messages) and dispatches them by invoking an appropriate handler. The performance and scalability of event-based systems come from the asynchronous decoupling of event handlers from the event source (e.g. the network interface) through the event loop, which enables many concurrent sessions to be serviced as a fine-grain sequential interleaving of actions within a single thread or a thread pool.

Our first example is a basic event loop that handles sessions of type

$$
\text { ?(Data).?(Data) . ! Result> }
$$

which says: at this side of the session, we first receive (?) a message, a Java object of type Data, then receive another Data message, and finish by sending (!) a Result. The other side will conform to the dual protocol, ! <Data $\rangle$. ! $\langle$ Data $\rangle$.? (Result).

We implement an event-driven server for handling multiple, concurrent sessions of the above type. The standard event loop pattern is adapted to SJ session programming as follows. The server registers the initialised session sockets with a session event selector (a session typed version of e.g. the Java NIO Selector [17]) to monitor them for event occurrences. By session typing, the first event on a new session will be the receipt of a Data message. After handling this event, the session socket is returned to the selector to await the second Data message. The functionality of the selector combines that of a dynamic collection for session sockets with asynchronous event detection. The key point, with respect to session typing, is that the selector is required to store not only sessions of the initial type (1), but also the intermediary type (2) ? (Data). ! <Result>.

Figure 1 outlines an ESJ program for the above server. Lines 1-2 declare a session set type containing session types (1) and (2). Lines 3-4 then declare and initialise the session event selector sel, an object of type SJSelector\{pSelector\}. This means sel can store and monitor session sockets of the two types in the pSelector set type. In SJ, the using statement has two main purposes. As in C\#, the declared resources (sel) are cleaned up after we leave the scope of the statement. In addition, session type checking requires the session type to be completed within the using, as we describe below.

The initial sessions can be registered with the selector as on Line 5. The main event loop then starts on Line 7. The first action in the loop is the select operation on Line 8, 


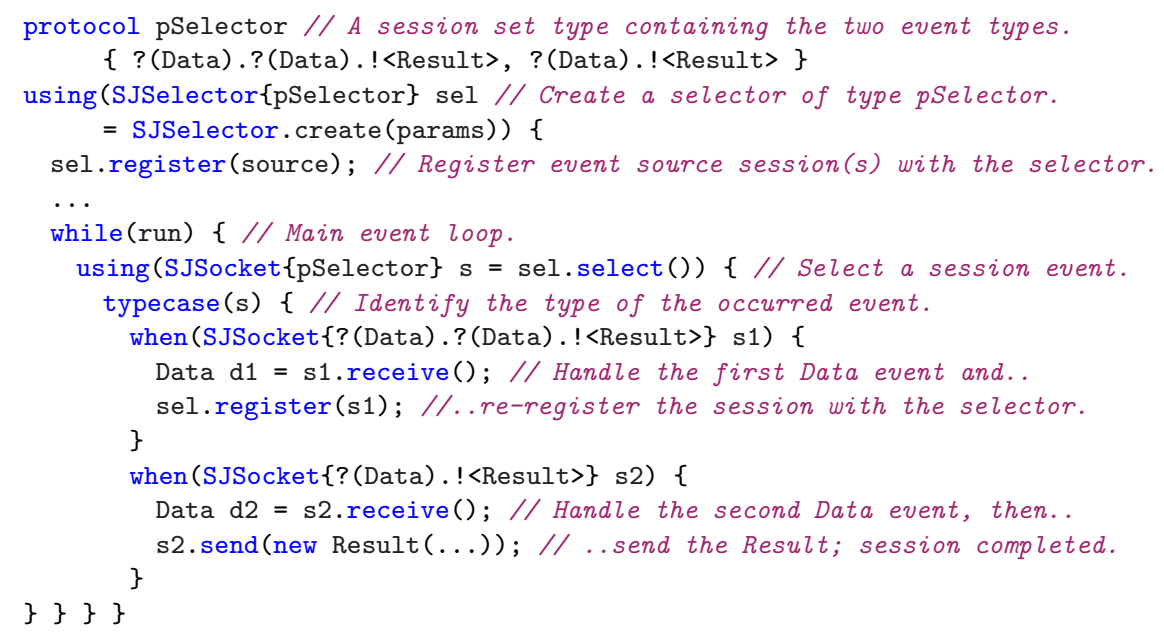

Fig. 1. Combining a session typed event selector with session typecase in a basic ESJ event loop.

which blocks until the selector detects an event occurrence on one of the registered session sockets. The returned session socket has type SJSocket\{pSelector\} - we know only that the session is of either of the two pSelector types - and is assigned to the variable s (enclosed by another using statement). To determine which event has occurred, i.e. whether we have received the first or second Data message on s, we use the session typecase starting on Line 9. The typecase selects the first when case for which the specified type matches the current runtime session type of the session. Static type checking ensures that the typecase covers all the cases of the pSelector set type, so at least one case is guaranteed to match at runtime. The when case on Lines 10-13 handles the first Data event, i.e. for session type (1). The session socket is rebound ${ }^{1}$ to the variable $\mathrm{s} 1$ of type SJSocket $\{!\langle$ Data $\rangle . !\langle$ Data $\rangle$.? (Result) $\}$, and $\mathrm{s} 1$ is used to receive the Data message on in Line 11. Since the second Data may not yet have arrived, Line 12 re-registers s1 with the selector (and then we loop to handle the next event). Similarly, the other when case on Lines 14-17 handles the second Data when the runtime session type of $\mathrm{s}$ is (2): following the session type, we receive the Data message and then send a Result via s2. The session is now finished; we do not re-register the session socket.

Event streams. In this example, we implement a prevalent pattern where an event handling party consumes streams of mixed events. This example extends the basic event loop to support session initiation and branching events, and uses session recursion to represent unbounded streams. We specify a simple event stream as sbegin.pStream (sbegin represents the server-side session initiation action), with pStream declared as

$$
\text { protocol pStream rec X [ ?\{NEXT: ?(Data).\#X, QUIT: \} ] }
$$

where $r e c \mathrm{X}[\ldots]$ binds the recursion type variable $\mathrm{X}$ within the scope of the brackets. Inside the recursion, the branch type $?\{\ldots\}$ allows the opposing session party to select

\footnotetext{
${ }^{1}$ typecase variable rebinding comes from dynamic typing in the $\lambda$-calculus [1], and ensures type soundness. The following "event streams" example further demonstrates this point.
} 


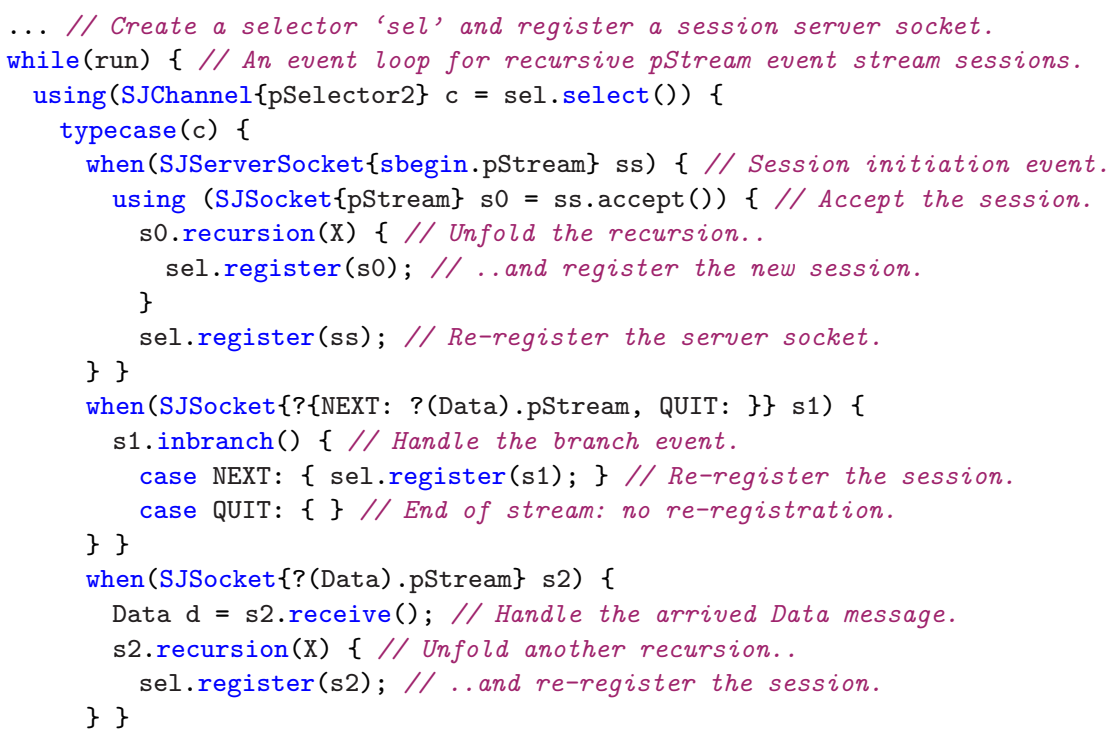

Fig. 2. Handling a stream of mixed event types, including session initiation and branching events.

one of the two paths, labelled NEXT and QUIT. If NEXT is selected, we receive a Data and the recursion is enacted (denoted by \#X). QUIT ends the stream, and the session is completed. For our ESJ implementation, we declare the session set type pSelector2

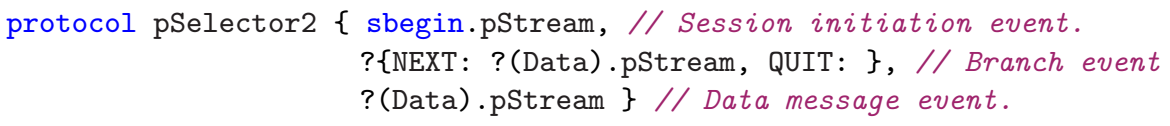

which specifies three event types: the session initiation event that creates the stream, the branch event when a branch label is received, and the message event when a Data arrives. Figure 2 lists an event loop for the above event stream. The new features in this program are as follows. The session initiation event described by sbegin.pStream involves calling accept on the SJServerSocket (a session-typed server endpoint for accepting client requests) $[25, \S 2]$ registered with the selector. Hence, the select operation returns an object of class SJChannel, the common superclass of SJSocket and SJServerSocket. Again, the type of the occurred event is determined using typecase, which rebinds (i.e. casts) the SJChannel to the appropriate subclass: SJServerSocket in the first when case, which handles the initiation event, and SJSocket in the other two cases. The recursive session is unfolded using the recursion construct, e.g. on Line 7, pStream is unfolded to ?\{NEXT: ? (Data).pStream, QUIT: \}. The branch type is implemented by the inbranch construct (Lines 13-16), which receives a label and selects the corresponding case; static typing ensures all specified cases are covered. 
Benefits due to session types. The main source of difficulty in traditional event-driven programming is the fragmentation of control flow across disjoint event handlers [2,29]. Below we summarise how session types counteract this key issue.

(1) Delineation of control flow. A session type is an abstraction of control flow (sequencing, branching and recursion) for interactions. Using session types, events are precisely defined by both the immediate action (e.g. the first ? (Data) in the first example) and by the remaining session flow (? (Data). ! <Result $>$ ). Traditional events lack the latter information, often requiring burdensome manual state management [2] to distinguish ambiguous events (e.g. the first and second Data messages). The combination of session set types and session typecase promotes clear structuring of event-driven programs, elucidating their communication and event-handling behaviour.

(2) Event-handling safety and progress. In addition to the above programming benefits, session types provide formal safety guarantees which traditional event-driven programming lacks. Static session type checking ensures event-handling safety (Theorem 4.3): each event is correctly handled as directed by the governing session type. The type safety also entails that each session is either completed or re-registered with a selector by the handler, ensuring that each session flow is faithfully preserved across separate event handlers. Combined with the standard linearity of session channels (which prevents interference of events by other threads), we obtain a strong progress property for asynchronous event programming, event progress (Theorem 4.6).

\section{A Process Model for Eventful Sessions}

We formalise the key programming ideas introduced in $\S 2$ as a small process calculus. The calculus, which we call ESP (Eventful Session Pi-calculus), is the $\pi$-calculus with session primitives [14,23] based on asynchronous communication semantics [13], to which we add minimal extensions for event-driven session programming: the message arrival predicate, session typecase, and session set types.

\subsection{Syntax of the Eventful Session $\pi$-Calculus}

Types. The type syntax of ESP extends the standard binary session types [14] with session set types. This simple extension allows us to treat type-safe event handing for an arbitrary collection of differently typed communication channels.

$$
\begin{array}{lll}
\text { (Shared) } & U::=\text { bool }|\langle S\rangle| \mathrm{X} \mid \mu \mathrm{X} . U \quad \text { (Value) } T::=U \mid\left\{S_{i}\right\}_{i \in I} \\
\text { (Session) } & S::=!(T) ; S|?(T) ; S| \oplus\left\{l_{i}: S_{i}\right\}_{i \in I}\left|\&\left\{l_{i}: S_{i}\right\}_{i \in I}\right| \mu \mathrm{X} . S|\mathrm{X}| \text { end }
\end{array}
$$

The shared types $\left(U, U^{\prime}, \ldots\right)$ are the booleans bool (we also use nat in examples), shared channel types $\langle S\rangle$ (shared channels of this type are used to establish sessions whose accepting side acts as $S$ ), type variables $(\mathrm{X}, \mathrm{Y}, \mathrm{Z}, \ldots)$ and recursive types. The session types $(S, S, \ldots)$ are standard $[14,23]$. The output type $!(T) ; S$ represents the output of a value of type $T$ followed by the behaviour represented by $S$; similarly for the dual input type ?(T);S. The select type $\oplus\left\{l_{i}: S_{i}\right\}_{i \in I}$ describes a behaviour that selects one of the labels $l_{i}$ followed by $S_{i}$. The branch type $\&\left\{l_{i}: S_{i}\right\}_{i \in I}$ waits for a select decision with $I$ options, and behaves as the $S_{i}$ corresponding to the chosen $l_{i}$. We assume recursive 


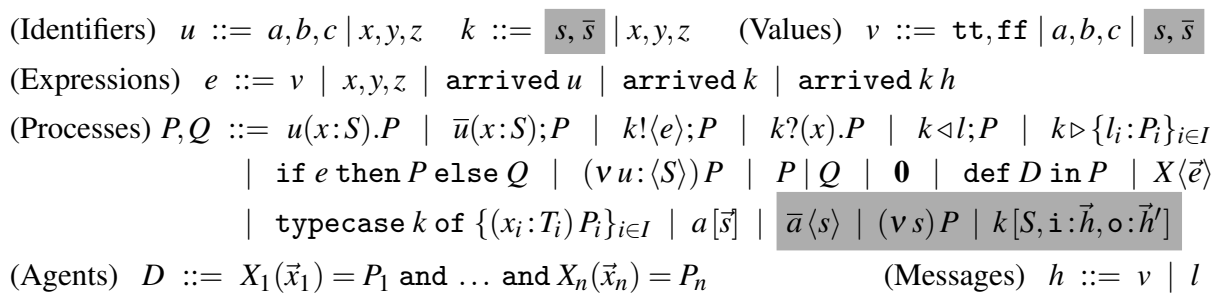

Fig. 3. The syntax of ESP processes.

types $\mu$ X.S are contractive, i.e. that type variables are guarded in the standard way. end represents session completion and is often omitted.

Value types for message values are the shared types and the session set types $\left\{S_{i}\right\}_{i \in I}$, where $I$ is finite (can be empty) and all $S_{i}$ are closed (i.e. do not contain free type variables). A session set type $\left\{S_{i}\right\}_{i \in I}$ represents a behaviour capable of safely interacting as any one of $S_{i}$. For example, a session with type $\{!($ bool),?(nat) $\}$ can safely interact with a session of both ?(bool) and !(nat) types. Session set types are used to type the typecase, and are so called for their set-like properties (derived from subtyping, $\S 4.1$ ), e.g. $\left\{S_{1}, S_{2}\right\}=\left\{S_{2}, S_{1}\right\}$ and $\{S, S\}=\{S\}$. A singleton $\{S\}$ is often written $S$. We write $\mathscr{T}$ for the set of all closed types, and $\mathscr{S}$ for the set of closed session types.

Processes. Figure 3 gives the syntax for ESP processes. Terms that only appear at runtime are shaded ; the other terms are user syntax. The new primitives are the message arrival predicate arrived for non-blocking inspection of messages buffers, and the session typecase typecase $k$ of $\{\ldots\}$ for dynamically inspecting the runtime type of a session. We also introduce asynchronous session initiation (cf. [14]).

Values $v, v^{\prime}, \ldots$ include the constants, shared channels $a, b, c, \ldots$, and session channels $s, s^{\prime}, \ldots$ A session channel $s$ designates one endpoint of a session, and $\bar{s}$ the opposing end of the same session, with $\overline{\bar{s}}=s^{2}$ Branch labels range over $l, l^{\prime}, \ldots$, variables over $x, y, z$, and process variables over $X, Y, Z$. Shared channel identifiers $u, u^{\prime}$ are shared channels and variables; session identifiers $k, k^{\prime}$ are session channels and variables. A session message $h$ is a value or a label. Expressions $e$ are values, variables, and the message arrival predicate: arrived $u$ for session initiation requests, arrived $k$ for session messages, and arrived $k h$, which checks for the arrival of the specific message $h$ at $k$. We write $\vec{s}$ and $\vec{h}$ for their respective vectors, and $\varepsilon$ for the empty vector.

The session initiation actions on shared channels are the request $\bar{u}(x: S) ; P$ and the accept $u(x: S) . P$. On an established session channel, output $k !\langle e\rangle ; P$ sends the value of $e$ through channel $k$, input $k$ ? $(x) . P$ receives a value through $k$, selection $k \triangleleft l ; P$ chooses and sends the label $l$ through $k$, and branching $k \triangleright\left\{l_{i}: P_{i}\right\}_{i \in I}$ follows the branch with the label received through $k$. The $(v u:\langle S\rangle) P$ binds a shared channel $u$ of type $\langle S\rangle$ to the scope of $P$. The session typecase typecase $k$ of $\left\{\left(x_{i}: T_{i}\right) P_{i}\right\}_{i \in I}$ takes a session channel $k$ and a list of cases $\left(x_{i}: T_{i}\right)$, each binding a free variable $x_{i}$ of type pattern $T_{i}$ in $P_{i}{ }^{3}$

\footnotetext{
${ }^{2}$ We simply say "session channel" rather than "session channel endpoints" (i.e. the programming entities used to perform session actions) for brevity; similarly for shared channels.

${ }^{3}$ The full typecase construct that supports typecase of general expressions $e$, matching variables in type patterns, and the default case is given in the long version available from [25].
} 


$$
\begin{aligned}
& \text { [Request1] } \quad \bar{a}(x: S) ; P \longrightarrow(v s)(P\{\bar{s} / x\} \mid \bar{s}[S, \text { i: }: \varepsilon, \circ: \varepsilon] \mid \bar{a}\langle s\rangle)(s \notin \operatorname{fn}(P)) \\
& \text { [Request2] } \quad a[\vec{s}] \mid \bar{a}\langle s\rangle \longrightarrow a[\vec{s} \cdot s] \\
& \text { [Accept] } \quad a(x: S) . P|a[s \cdot \vec{s}] \longrightarrow P\{s / x\}| s[S, \mathrm{i}: \varepsilon, \circ: \varepsilon] \mid a[\vec{s}] \\
& \text { [Send] } \quad s !\langle v\rangle ; P|s[!(T) ; S, \circ: \vec{h}] \longrightarrow P| s[S, \circ: \vec{h} \cdot v] \\
& \text { [Receive] } \quad s ?(x) \cdot P|s[?(T) ; S, i: v \cdot \vec{h}] \longrightarrow P\{v / x\}| s[S, i: \vec{h}] \\
& \text { [Sel], [Bra] } \quad s \triangleleft l_{i} ; P\left|s\left[\oplus\left\{l_{i}: S_{i}\right\}_{i \in I}, \circ: \vec{h}\right] \longrightarrow P_{i}\right| s\left[S_{i}, \circ: \vec{h} \cdot l_{i}\right] \quad(i \in I) \\
& s \triangleright\left\{l_{j}: P_{j}\right\}_{j \in J}\left|s\left[\&\left\{l_{i}: S_{i}\right\}_{i \in I}, \dot{i}: l_{i} \cdot \vec{h}\right] \longrightarrow P_{i}\right| s\left[S_{i}, \dot{i}: \vec{h}\right] \quad(i \in I \cap J) \\
& \text { [Comm] } \quad s[0: v \cdot \vec{h}]\left|\bar{s}\left[\mathrm{i}: \vec{h}^{\prime}\right] \longrightarrow s[0: \vec{h}]\right| \bar{s}\left[\mathrm{i}: \vec{h}^{\prime} \cdot v\right] \\
& \text { [Instance] } \quad \operatorname{def} D \text { in }(X\langle\vec{v}\rangle \mid Q) \longrightarrow \operatorname{def} D \text { in } P\{\vec{v} / \vec{x}\} \mid Q \quad X(\vec{x})=P \in D \\
& \text { [Arriv-req] } \quad E[\operatorname{arrived} a]|a[\vec{s}] \longrightarrow E[b]| a[\vec{s}] \quad(|\vec{s}| \geq 1) \downarrow b \\
& \text { [Arriv-msg] } \quad E[\text { arrived } s h]|s[\mathrm{i}: \vec{h}] \longrightarrow E[b]| s[\mathrm{i}: \vec{h}] \quad\left(\vec{h}=h \cdot \vec{h}^{\prime}\right) \downarrow b
\end{aligned}
$$

Fig. 4. Selected reduction rules.

Our calculus incorporates two forms of asynchronous communication, asynchronous session initiation [18] and asynchronous session communication (over an established session). The former models the unordered transport of session request messages to acceptors listening on a shared channel. We use $\bar{a}\langle s\rangle$ to represent a request message in transit on shared channel $a$, carrying a fresh session channel $s$ of type $S$. In real network communications, messages are buffered for reading on arrival at the destination. This mechanism is formalised by introducing a shared input queue $a[\vec{s}]$, which represents an acceptor's input buffer at $a$ containing the pending requests for sessions $\vec{s}$.

Communication in an established session is asynchronous but order-preserving, as in TCP. For this purpose, each session channel $s$ is associated with an endpoint configuration (or simply configuration) $s\left[S, i: \vec{h}, \circ: \vec{h}^{\prime}\right]$, which encapsulates both input (i) and output (o) buffers. Sending a message first enqueues it at the source o-buffer before it is eventually transferred to the destination i-buffer, signifying the arrival of that message. For both unordered session requests and ordered session messages, decoupling message transmission and arrival captures the intuitive semantics for arrived: only messages that are present in the local input buffer can be detected, and not those still in transit. The $S$ in $s\left[S, i: \vec{h}, \circ: \vec{h}^{\prime}\right]$ is called the active type, and represents the remaining session actions to be performed at this endpoint (representing a runtime session monitoring mechanism). For brevity, one or more components may be omitted from a configuration when they are irrelevant, written as e.g. $s[S]$ or $s[i: \vec{h}]$.

$(v s) P$ binds both session endpoints, $s$ and $\bar{s}$, making them private within $P$. The remaining constructs, conditional, parallel composition, agent definition and instantiation, and inaction, are standard. Type annotations and $\mathbf{0}$ are often omitted. The notions of free variables and channels are standard [23]; we write $\mathrm{fn}(P)$ for the set of free channels in $P$. Terms in closed user syntax are called programs. 


\subsection{Operational Semantics}

The reduction relation on closed terms $\longrightarrow$ captures the communication and event handling dynamics of ESP processes, and updates active types as session interactions progress. Figure 4 lists the key rules. We use the standard evaluation contexts $E$ [-] defined as $E::=-|s !\langle E\rangle ; P|$ if $E$ then $P$ else $Q \mid X\langle\vec{v} E \vec{e}\rangle$. Structural congruence $\equiv$ and the omitted reduction rules are standard; the full definitions are found at [25].

[Request1] issues a new request for a session of type $S$ via shared channel $a$. A fresh (i.e. $v$-bound) session with endpoints $s$ (acceptor-side) and $\bar{s}$ (requestor-side) and the initial configuration at the requestor are generated, dispatching the session request message $\bar{a}\langle s\rangle$. [Request2] enqueues the request in the shared input queue at $a$. [Accept] dequeues the earliest session request, instantiates the session to the $s$ in the request message, and creates the acceptor-side configuration: the new session is now established.

[Send] enqueues a value in the o-buffer of the local configuration and removes the prefix from the current active type, signifying the completion of this action. [Receive] dequeues the first value from the i-buffer of the local configuration and updates the active type accordingly. [Sel] and [Bra] similarly enqueue and dequeue a label, using the label to select the appropriate case in the current active type. Note these rules manipulate only the local configurations, and output actions are always non-blocking. The actual transmission of a session message is embodied by [Comm], which removes the first message from the o-buffer of the source configuration and enqueues it in the i-buffer at the opposing configuration. [Instance] is a standard recursion rule for processes.

Although input actions block if no message is available in the corresponding input buffer, blocking can be avoided using the message arrival predicates. [Arriv-req] evaluates arrived $a$ to tt if the queue is non-empty; similarly for arrived $k$ (rule omitted). [Arriv-msg] evaluates arrived $s h$ to tt if the queue is non-empty and the first message matches $h$. The notation $e \downarrow b$ means $e$ evaluates to the boolean value $b$. Lastly, [Typecase] is the key rule which enables dynamic inspection of the active type of a session. The process continues the session $s$ along the first $P_{i}$ for which $T_{i}$ can be successfully matched against the current active type $S$ up to subtyping (defined in $\S 4.1$ ).

\subsection{Representing High-level Event Constructs in ESP}

Example 3.1 (Selector and Event Loops). The core functionality of SJSelector (illustrated in $\S 2$ ) can be distilled to three operations: create a new selector, register a channel with the selector, and select (i.e. retrieve from the selector) a channel on which a message has arrived. Session typecase is then used to type the selected channel. We extend ESP with these operations, with the following reduction semantics. ${ }^{4}$ We omit type annotations for selectors, which we shall discuss in $\S 4$.

$$
\begin{aligned}
& \text { new sel } r \text { in } P \longrightarrow(v r)(P \mid \operatorname{sel}\langle r, \varepsilon\rangle) \quad \text { reg } s \text { to } r \text { in } P|\operatorname{sel}\langle r, \vec{s}\rangle \longrightarrow P| \operatorname{sel}\langle r, \vec{s} \cdot s\rangle \\
& \operatorname{select}(r)\left\{\left(x_{i}: T_{i}\right): P_{i}\right\}_{i \in I}|\operatorname{sel}\langle r, s \cdot \vec{s}\rangle| s[S, i: \vec{h}] \longrightarrow P_{i}\left\{s / x_{i}\right\}|\operatorname{sel}\langle r, \vec{s}\rangle| s[S, i: \vec{h}] \quad(\vec{h} \neq \varepsilon) \\
& \operatorname{select}(r)\left\{\left(x_{i}: T_{i}\right): P_{i}\right\}_{i \in I}|\operatorname{sel}\langle r, s \cdot \vec{s}\rangle| s[i: \varepsilon] \longrightarrow \operatorname{select}(r)\left\{\left(x_{i}: T_{i}\right): P_{i}\right\}_{i \in I}|\operatorname{sel}\langle r, \vec{s} \cdot s\rangle| s[i: \varepsilon]
\end{aligned}
$$

\footnotetext{
${ }^{4}$ The selector semantics presented here is based on polling, which is suitable for our current purpose (i.e. giving a semantic basis for selectors). For further discussion on the behaviour and implementation of selectors, see $\S 5.2$.
} 
In the second line, $S$ and $T_{i}$ satisfy the condition for [Typecase] in Figure 4. We also add structural rules, e.g. $(v r) \operatorname{sel}\langle r, \varepsilon\rangle \equiv \mathbf{0}$. Operator new sel $r$ in $P$ (binding $r$ in $P$ ) creates a new selector sel $\langle r, \varepsilon\rangle$, named $r$, with the empty queue $\varepsilon$. reg $s$ to $r$ in $P$ registers the session channel with $r$, adding $s$ to the queue $\vec{s}$. select $(r)\left\{\left(x_{i}: T_{i}\right): P_{i}\right\}_{i \in I}$ checks whether a message is available (i.e. an event has occurred) on the first session in the queue, $s$. If so, we select the first $P_{i}$ for which the type of $s$ matches $T_{i}$ (condition omitted); otherwise, $s$ is re-enqueued and the next session is tested.

We now show this behaviour can be easily encoded by combining the message arrival predicate and session typecase. We again omit type annotations (until $\S 4$ ).

$$
\begin{aligned}
& \text { [new sel } r \text { in } P \rrbracket \stackrel{\text { def }}{=}(v b)(\bar{b}(\bar{r}) ; b(r) .[[P] \mid b:[\varepsilon]) \quad \text { [reg } s \text { to } r \text { in } P \rrbracket \stackrel{\text { def }}{=} \bar{r} !\langle s\rangle ;[[P] \\
& \left.\llbracket\left[\operatorname{select}(r)\left\{\left(x_{i}: S_{i}\right): P_{i}\right\}_{i \in I}\right] \stackrel{\text { def }}{=} \quad \llbracket \operatorname{sel}\left\langle r, \vec{s} \cdot \vec{s}^{\prime}\right\rangle\right] \stackrel{\text { def }}{=} \vec{r}\left[0: \vec{s}^{\prime}\right] \mid r[i: \vec{s}] \\
& \operatorname{def} \operatorname{Select}(x \bar{x})=x ?(y) \text {; if arrived } y \text { then typecase } y \text { of }\left\{\left(x_{i}: T_{i}\right):\left[\left[P_{i}\right]\right]\right\}_{i \in I} \\
& \text { else } \bar{x} !\langle y\rangle ; \text { Select }\langle x \bar{x}\rangle \quad \text { in Select }\langle r \bar{r}\rangle
\end{aligned}
$$

The use of arrived is the key to avoiding blocked inputs, allowing the selector to proceed asynchronously while handling any available messages. The operations on the collection queue (via $r$ and $\bar{r}$ ) exchange session channels, hence session delegation [14] is essential. We can easily check that this encoding is operationally faithful to the native selector (i.e. the direct ESP extension) using a suitable bisimulation. Using the above selector, a basic event loop similar to Figure $1(\S 2)$ can be represented as:

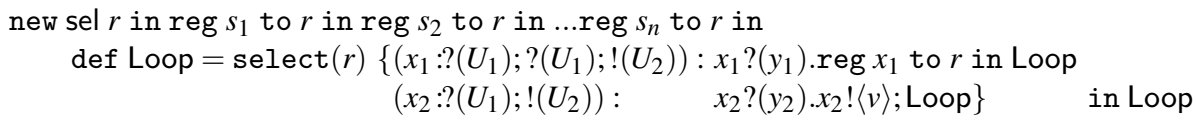

In $\S 4.4$, we prove event progress for processes that use selectors, such as event loops.

Example 3.2 (Switch-receive). Join patterns $[3,10]$ are one mechanism for correlating multiple event occurrences [8]. Programmers use join patterns as guards to handle particular combinations (i.e. conjunctions) of message arrivals on one or more sessions. The switch receive construct in Sing\# [9] implements this mechanism for channel contracts (a version of session types), which we can formalise as an ESP extension.

$$
\text { switch-receive }\left\{J_{1}: P_{1}, \ldots, J_{m}: P_{m}\right\} \quad J_{j}::=s_{j 1} . l_{j 1}\left(x_{j 1}: U_{j 1}\right) \wedge \ldots \wedge s_{j n_{j}} \cdot l_{j n_{j}}\left(x_{j n_{j}}: U_{j n_{j}}\right)
$$

Above we set $m, n, j \geq 1$ and all $s_{j 1}, . ., s_{j n_{j}}$ should be pairwise distinct for each $j$. Each $J_{j}$ denotes a join pattern, a conjunction of expressions of the form $s_{j i} . l_{j i}\left(x_{j i}: U_{j i}\right)$, where $l_{j i}$ is a branch label expected at $s_{j i}$, and $\left(x_{j i}: U_{j i}\right)$ is a formal parameter for a message of type $U_{j i}$ following $l_{j i}$. The formal semantics of switch-receive can be found at [25]; here, we informally illustrate its behaviour through a simple example. Let $R$ be:

$$
R \stackrel{\text { def }}{=} \text { switch-receive }\left\{s . l_{1}\left(x_{1}\right): P, s . l_{2}\left(x_{2}\right) \wedge s^{\prime} \cdot l_{3}\left(x_{3}\right): Q\right\}
$$

$R$ listens on $s$ (where $l_{1}$ or $l_{2}$ is expected) and $s^{\prime}$ (for $l_{3}$ ); by the above design, a message is expected to follow each label. Suppose $l_{1}$ and then $v_{1}$ arrive: $R$ will become $P\left\{v_{1} / x_{1}\right\}$. On the other hand, if $l_{2}$ and $v_{2}$ arrive at $s$, and $v_{2}$ and $l_{3}$ and $v_{3}$ at $s^{\prime}$, then $R$ becomes $Q\left\{v_{2} v_{3} / x_{2} x_{3}\right\}$. In all other cases, $R$ continues to wait for further messages on $s$ and $s^{\prime}$.

An inductive ESP encoding of switch-receive can be formulated using arrived. We illustrate the idea of the encoding using $R$ from above. Assuming that the communication of a branch label is always followed by a message: def SRLoop $=$ 


$$
\begin{array}{ll}
\text { if } \quad\left(\operatorname{arrived} s l_{1}\right) & \text { then } s \triangleright l_{1}: s ?\left(x_{1}\right) \cdot[[P] \\
\text { else if }\left(\operatorname{arrived} s l_{2} \text { and arrived } s^{\prime} l_{3}\right) & \text { then } s \triangleright l_{2}: s ?\left(x_{2}\right) \cdot s^{\prime} \triangleright l_{3}: s^{\prime} ?\left(x_{3}\right) .[[Q]] \\
& \text { else SRLoop }
\end{array}
$$

Above, the sequential branch notation $s_{i} \triangleright l_{i}: P$ stands for a branch at $s_{i}$ that omits the superfluous branches ruled out by the preceding arrived. More complex join patterns featuring predicates on received values are also encodable into ESP.

\section{Typing Eventful Sessions}

This section presents a type discipline for ESP and establishes its key properties: subtyping (Proposition 4.1); type safety (Theorem 4.2); communication and event-handling safety (Theorem 4.3); soundness of the ESP encoding of a high-level event primitive, selector (Proposition 4.4); and event progress (Theorem 4.6).

\subsection{Subtyping from Composability}

If $P$ has a session channel $s$ of type $S$, the ways in which $P$ can use $s$ are at most as $S$; e.g. if $S$ is $\&\left\{l_{1}: S_{1}, l_{2}: S_{2}\right\}$, then $P$ handles labels $l_{1}$ and $l_{2}$ but not any others, thus $P$ can interact with peers that select either one of these two labels. By this intuition, for a process $Q$ with session type $S^{\prime}$ to be safely used in place of $P$ (i.e. $S^{\prime} \leq S$ ), $Q$ should be composable in the same or more ways (i.e. with more peers) than $P$; e.g. if $S^{\prime}$ is $\&\left\{l_{i}: S_{i}\right\}_{1 \leq i \leq 3}$, then $Q$ can also interact with peers that select $l_{3}$. Formally, the subtyping relation is defined on the set of all closed and contractive types $\mathscr{T}$ as follows: $T$ is a subtype of $T^{\prime}$, written $T \leq T^{\prime}$, if $\left(T, T^{\prime}\right)$ is in the largest fixed point of the monotone function $\mathscr{F}: \mathscr{P}(\mathscr{T} \times \mathscr{T}) \rightarrow \mathscr{P}(\mathscr{T} \times \mathscr{T})$, where $\mathscr{F}(\mathscr{R})$ is given by:

$\{($ bool, bool $)\} \cup\left\{\left(\langle S\rangle,\left\langle S^{\prime}\right\rangle\right) \mid\left(S, S^{\prime}\right),\left(S^{\prime}, S\right) \in \mathscr{R}\right\}$

$$
\begin{aligned}
& \cup\left\{\left(\mu \mathrm{X} . U, U^{\prime}\right) \mid\left(U\{\mu \mathrm{X} . U / \mathrm{X}\}, U^{\prime}\right) \in \mathscr{R}\right\} \cup\left\{\left(U, \mu \mathrm{X} . U^{\prime}\right) \mid\left(U, U^{\prime}\left\{\mu \mathrm{X} . U^{\prime} / \mathrm{X}\right\}\right) \in \mathscr{R}\right\} \\
& \cup\left\{\left(!\left(T_{1}\right) ; S_{1}^{\prime}, !\left(T_{2}\right) ; S_{2}^{\prime}\right) \mid\left(T_{2}, T_{1}\right),\left(S_{1}^{\prime}, S_{2}^{\prime}\right) \in \mathscr{R}\right\} \cup\left\{\left(?\left(T_{1}\right) ; S_{1}^{\prime}, ?\left(T_{2}\right) ; S_{2}^{\prime}\right) \mid\left(T_{1}, T_{2}\right),\left(S_{1}^{\prime}, S_{2}^{\prime}\right) \in \mathscr{R}\right\} \\
& \cup\left\{\left(\oplus\left\{l_{i}: S_{i}\right\}_{i \in I}, \oplus\left\{l_{j}: S_{j}^{\prime}\right\}_{j \in J}\right) \mid \forall i \in I \subseteq J .\left(S_{i}, S_{i}^{\prime}\right) \in \mathscr{R}\right\} \\
& \cup\left\{\left(\&\left\{l_{i}: S_{i}\right\}_{i \in I}, \&\left\{l_{j}: S_{j}^{\prime}\right\}_{j \in J}\right) \mid \forall j \in J \subseteq I .\left(S_{j}, S_{j}^{\prime}\right) \in \mathscr{R}\right\} \\
& \cup\left\{\left(\mu \mathrm{X} . S, S^{\prime}\right) \mid\left(S\{\mu \mathrm{X} . S / \mathrm{X}\}, S^{\prime}\right) \in \mathscr{R}\right\} \cup\left\{\left(S, \mu \mathrm{X} . S^{\prime}\right) \mid\left(S, S^{\prime}\left\{\mu \mathrm{X} . S^{\prime} / \mathrm{X}\right\}\right) \in \mathscr{R}\right\} \\
& \cup\left\{\left(\left\{S_{i}\right\}_{i \in I},\left\{S_{j}^{\prime}\right\}_{j \in J}\right) \mid \neg(|I|=|J|=1), \forall j \in J, \exists i \in I .\left(S_{i}, S_{j}^{\prime}\right) \in \mathscr{R}\right\}
\end{aligned}
$$

Line 1 is standard: $\langle S\rangle$ is invariant on $S$ since it supports both $S$ and $\bar{S}$ (see duality below). Lines 2 and 6 are the standard rules for recursion. In Line 3, the linear output (resp. input) is contravariant (resp. covariant) on the message type following [23]. In Line 4, a select that requires support for more labels means fewer peers can be safely composed; dually for branching in Line 5. Finally, the ordering of set types says that if every element in the set type $\left\{S_{j}^{\prime}\right\}_{j \in J}$ has a subtype in $\left\{S_{i}\right\}_{i \in I}$, then the latter is at least as composable as the former. The condition $\neg(|I|=|J|=1)$ avoids the case where $\left\{S_{i}\right\}_{i \in I}=S$ and $\left\{S_{j}^{\prime}\right\}_{j \in J}=S^{\prime}$, which would make the relation universal.

We now clarify the semantics of $\leq$ using duality. The dual of $S$, denoted $\bar{S}$, is defined in the standard way: $\overline{!(T) ; S}=?(T) ; \bar{S}, \overline{?(T) ; S}=!(T) ; \bar{S}, \overline{\mu \mathrm{X} . S}=\mu \mathrm{X} . \bar{S}, \overline{\mathrm{X}}=\mathrm{X}$, $\overline{\oplus\left\{l_{i}: S_{i}\right\}_{i \in I}}=\&\left\{l_{i}: \overline{S_{i}}\right\}_{i \in I}, \overline{\&\left\{l_{i}: S_{i}\right\}_{i \in I}}=\oplus\left\{l_{i}: \overline{S_{i}}\right\}_{i \in I}$ and $\overline{\text { end }}=$ end. The set of composable types of $\left\{S_{i}\right\}_{i \in I}$ is defined as: $\operatorname{comp}\left(\left\{S_{i}\right\}_{i \in I}\right)=\cup_{i \in I}\left\{S^{\prime} \mid S^{\prime} \leq \overline{S_{i}}\right\}$. We observe:

Proposition 4.1 (Subtyping Properties). (1) $\leq$ is a preorder; (2) given $T$ and $T^{\prime}, T \leq$ $T^{\prime}$ is decidable; and (3) (semantics of $\leq$ ) $T_{1} \leq T_{2}$ if and only if $\operatorname{comp}\left(T_{2}\right) \subseteq \operatorname{comp}\left(T_{1}\right)$. 


$$
\begin{aligned}
& \frac{\Gamma \vdash u:\langle S\rangle}{\Gamma \cdot u: T \vdash u: T} \text { (Shared) } \frac{\Gamma \text { (aReq) }}{\Gamma \vdash \operatorname{arrived} u: \text { bool }} \quad \frac{\Gamma \vdash \operatorname{arrived} k h: \text { bool }}{\Gamma, \Sigma \vdash \operatorname{arrived} k: \text { bool }} \text { (aMsg) } \\
& \frac{\Gamma \vdash v: U}{\Gamma, \Sigma \cdot k: ?(U) ; S \vdash \text { arrived } k v: \text { bool }}(\text { aVal }) \quad \frac{l \in\left\{l_{i}\right\}_{i \in I}}{\Gamma, \Sigma \cdot k: \&\left\{l_{i}: S_{i}\right\}_{i \in I} \vdash \text { arrived } k l: \text { bool }} \text { (aLab) } \\
& \frac{\Gamma \vdash P \triangleright \Sigma^{\prime} \quad \Sigma^{\prime} \leq \Sigma}{\Gamma \vdash P \triangleright \Sigma} \text { (Subs) } \frac{\Gamma \vdash u:\langle S\rangle \quad \Gamma \vdash P \triangleright \Sigma \cdot x: S}{\Gamma \vdash u(x: S) \cdot P \triangleright \Sigma}(\text { Acc }) \frac{\Gamma \vdash u:\langle S\rangle \quad \Gamma \vdash P \triangleright \Sigma \cdot x: \bar{S}}{\Gamma \vdash \bar{u}(x: \bar{S}) ; P \triangleright \Sigma} \text { (Req) } \\
& \frac{\Gamma, \Sigma \vdash e: U \quad \Gamma \vdash P \triangleright \Sigma \cdot k: S}{\Gamma \vdash k !\langle e\rangle ; P \triangleright \Sigma \cdot k: !(U) ; S} \text { (Send) } \quad \frac{\Gamma \cdot x: U \vdash P \triangleright \Sigma \cdot k: S}{\Gamma \vdash k ?(x) \cdot P \triangleright \Sigma \cdot k: ?(U) ; S}(\text { Recv) } \\
& \frac{\Gamma \vdash P \triangleright \Sigma \cdot k: S}{\Gamma \vdash k !\left\langle k^{\prime}\right\rangle ; P \triangleright \Sigma \cdot k: !(T) ; S \cdot k^{\prime}: T} \text { (SSend) } \quad \frac{\Gamma \vdash P \triangleright \Sigma \cdot k: S \cdot x: T}{\Gamma \vdash k ?(x) \cdot P \triangleright \Sigma \cdot k: ?(T) ; S} \text { (SRecv) } \\
& \frac{1 \leq i \leq n \quad \Gamma \vdash P \triangleright \Sigma \cdot k: S_{i}}{\Gamma \vdash k \triangleleft l_{i} ; P \triangleright \Sigma \cdot k: \oplus\left\{l_{i}: S_{i}\right\}_{i \in I}} \text { (Select) } \quad \frac{\forall i .1 \leq i \leq n \quad \Gamma \vdash P_{i} \triangleright \Sigma \cdot k: S_{i}}{\Gamma \vdash k \triangleright\left\{l_{i}: P_{i}\right\}_{n} \triangleright \Sigma \cdot k: \&\left\{l_{i}: S_{i}\right\}_{i \in I}} \text { (Branch) } \\
& \frac{\Gamma, \Sigma \vdash e \text { : bool } \Gamma \vdash P \triangleright \Sigma \Gamma \vdash Q \triangleright \Sigma}{\Gamma \vdash \text { if } e \text { then } P \text { else } Q \triangleright \Sigma} \text { (If) } \frac{\Gamma \cdot a:\langle S\rangle \vdash P \triangleright \Sigma \cdot a}{\Gamma \vdash(v a:\langle S\rangle) P \triangleright \Sigma} \text { (Chan) } \frac{\Gamma \vdash P \triangleright \Sigma \quad \Gamma \vdash Q \triangleright \Sigma^{\prime}}{\Gamma \vdash P \mid Q \triangleright \Sigma \cdot \Sigma^{\prime}} \text { (Par) } \\
& \frac{\forall i \in I \quad \Gamma \vdash P_{i} \triangleright \Sigma \cdot x_{i}: T_{i} \quad \cup_{i \in I} T_{i} \leq T}{\Gamma \vdash \text { typecase } k \text { of }\left\{\left(x_{i}: T_{i}\right) P_{i}\right\}_{i \in I} \triangleright \Sigma \cdot k: T} \text { (Typecase) } \frac{\Sigma \text { end only }}{\Gamma \vdash a[\varepsilon] \triangleright \Sigma \cdot a} \text { (Queue) } \frac{\Sigma \text { end only }}{\Gamma \vdash \mathbf{0} \triangleright \Sigma} \text { (Nil) }
\end{aligned}
$$

Fig. 5. Selected typing rules for ESP programs.

\subsection{Program Typing}

We first define a typing system for programs (§3.1). Program typing, which can be considered a static typing phase performed by a compiler on user-level code before execution, uses two environments:

$$
\Gamma::=\emptyset|\Gamma \cdot u: U| \Gamma \cdot X: \vec{T} \quad \Sigma::=\emptyset|\Sigma \cdot a| \Sigma \cdot k:\left\{S_{i}\right\}_{i \in I}
$$

$\Gamma$ is called the shared environment, which maps variables and shared channels to constant types and process variables to sequences of message types; $\Sigma$ is called the linear environment, which maps session channels to set types (writing $k: S$ for $k:\{S\}$ ) and records the shared channels that have input queues (to ensure that each $a$ has exactly one queue). $\Sigma \cdot a$ means $a \notin \operatorname{dom}(\Sigma)$, and similarly for others. Subtyping is extended to environments by $\Sigma \leq \Sigma^{\prime}$ iff $\operatorname{dom}(\Sigma)=\operatorname{dom}\left(\Sigma^{\prime}\right)$ and $\forall k \in \operatorname{dom}(\Sigma) \cdot \Sigma(k) \leq \Sigma^{\prime}(k)$. The typing judgements for processes and expressions are:

$$
\Gamma \vdash P \triangleright \Sigma \quad \Gamma, \Sigma \vdash e: T
$$

On the left, the program typing judgement says the program $P$, under shared environment $\Gamma$, features the channel usage specified by linear environment $\Sigma$; similarly for the expression typing judgement, which can be shortened to $\Gamma \vdash e: T$ if $\Sigma$ is not required.

Figure 5 presents selected typing rules for programs (the rules can be seen in full at [25]). (Shared) is the standard rule for shared channel expressions (the Figure omits the other standard expression typing rules, e.g. $\Gamma \vdash \mathrm{tt}$ : bool, $\Gamma \cdot x$ : bool $\vdash x$ : bool, etc.). The next four rules check that the message arrival predicate is used appropriately. (aReq) is for session request arrival on shared channels. (aVal) and (aLab) are respectively for the arrival of a specific value and branch label on a session channel; (aMsg) is for either kind and any value of session message. (Subs) is standard subsumption. 
Although ESP has asynchronous session initiation and i/o-buffered communication semantics, program typing for the basic session initiation and communication actions remain standard. Rule (Acc) (resp. (Req)) says that the session implementation following an accept (resp. request) should conform to the type annotation and the shared channel type. (Send) and (Recv) check that the expected value types are communicated. For an output, we check the session type prefix is ! $(U)$ where $U$ is the message expression type; dually for input. Rules (SSend) and (SRecv) for session delegation are similar. (Select) checks that the selection action chooses and follows one of the specified branches; (Branching) checks that branching offers at least the specified branches. The (If) rule checks conditional expressions.

(Queue) records the presence of an empty shared input queue in the linear environment. (Par) disallows multiple queues for the same shared channel, and prevents the composition of processes with the same session (i.e. linear) channels. (Chan) records $a:\langle S\rangle$ in the shared environment after checking the presence of a (unique) shared queue for $a$. (Nil) and the omitted rules for agent definition and instantiation are standard from [14]. " $\Sigma$ end only" means $\forall k \in \operatorname{dom}(\Sigma), \Sigma(k)=$ end. Finally, (Typecase) is an extension of dynamic types in the $\lambda$-calculus [1] to session types. It checks for each case that the body $P_{i}$ is typable under $\Sigma$ with the session channel $k$ "rebound" to $x_{i}$ as type $T_{i}$. Then the whole process is typed with $k$ set to $T$, which is a supertype of all $T_{i}$.

\subsection{Type Soundness and Event-Handling Safety}

This subsection establishes the fundamental safety properties of ESP program typing. The proofs require additional runtime process typing: we note here that our approach extends [23] to support the new configuration active types and the finer-grained i/obuffers, and the full details can be found at [25]. We start with type soundness.

Theorem 4.2 (Type Soundness). (1) If $\Gamma \vdash P \triangleright \Sigma$ and $P \equiv P^{\prime}$, then we have $\Gamma \vdash P \triangleright \Sigma$. (2) If $\Gamma \vdash P \triangleright \emptyset$ and $P \longrightarrow Q$, then we have $\Gamma \vdash Q \triangleright \emptyset$.

Next we prove communication safety. An s-redex is a parallel composition of two processes that has one of the following shapes:

(a) $s !\langle v\rangle ; P \mid s\left[!(T) ; S, \mathrm{i}: \vec{h}, \circ: \vec{h}^{\prime}\right]$

(c) $s ?(x) . P \mid s\left[?(T) ; S, \mathrm{i}: v \cdot \vec{h}, 0: \vec{h}^{\prime}\right]$

(e) $s\left[S, i: \vec{h}_{1}, \circ: v \cdot \vec{h}_{1}^{\prime}\right] \mid \bar{s}\left[S^{\prime}, \mathrm{i}: \vec{h}_{2}, \circ: \vec{h}_{2}^{\prime}\right]$

(f) $E[$ arrived $s v] \mid s\left[?(U) ; S, i: \vec{h}, \circ: \vec{h}^{\prime}\right]$ with $v$ of type $U$

(g) $E\left[\right.$ arrived $\left.s l_{j}\right] \mid s\left[\&\left\{l_{i}: S_{i}\right\}_{i \in I}, i: \vec{h}, \circ: \vec{h}^{\prime}\right]$ with $j \in I$

(h) typecase $s$ of $\left\{\left(x_{i}: T_{i}\right) P_{i}\right\}_{i \in I} \mid s[S]$ with $\exists i \in I . T_{i} \leq S$

All redexes require the immediate action to correspond with the active type prefix in the local configuration. ( $\mathrm{f}-\mathrm{h}$ ) are for the new primitives for asynchronous event handing. We say a process $P$ is an error if up to structural congruence (following $[15, \S 5]$ ), $P$ contains more than two $s$-processes which do not form an $s$-redex, or an expression in $P$ contains a type error in the standard sense. Then from Theorem 4.2 we obtain:

Theorem 4.3 (Communication and Event-Handling Safety). If $P$ is a well-typed program, then $\Gamma \vdash P \triangleright \emptyset$, and $P$ never reduces to an error. 


\subsection{Typing Event Selectors and Event Progress}

Typing selectors. Typing rules for the extended ESP selector construct defined in Example 3.1 naturally follow from the ESP-typing of the selector encoding (§3.3). We restore the previously omitted selector type annotations: new sel $\langle T\rangle r$ in $P$ creates a selector that stores channels of type $T$. Then the type for a user of the selector is written $\overline{\mathrm{sel}}(T)$, and for the selector itself $\operatorname{sel}(T)$. For simplicity, we assume these types do not occur as part of other types. The linear environment $\Sigma$ is extended with two additional type assignments, $r: \operatorname{sel}(T)$ and $r: \operatorname{sel}(T)$, the latter only used for runtime typing for selector queues. The program typing rules for the selector operations are:

$$
\begin{aligned}
& \frac{\Gamma \vdash P \triangleright \Sigma \cdot r: \overline{\operatorname{sel}}(T)}{\Gamma \vdash \text { new sel }\langle T\rangle r \text { in } P \triangleright \Sigma} \text { (Selector) } \quad \frac{\Gamma \vdash P \triangleright \Sigma \cdot r: \overline{\operatorname{sel}}(T) \quad S \leq T}{\Gamma \vdash \operatorname{reg} s \text { to } r \text { in } P \triangleright \Sigma \cdot r: \overline{\operatorname{sel}}(T) \cdot s: S} \text { (Reg) } \\
& \frac{\forall i \in I . \Gamma \vdash P_{i} \triangleright \Sigma \cdot r: \overline{\operatorname{sel}}(T) \cdot x_{i}: S_{i} \quad S_{i} \leq T}{\Gamma \vdash \operatorname{select}(r)\left\{\left(x_{i}: S_{i}\right): P_{i}\right\}_{i \in I} \triangleright \Sigma \cdot r: \overline{\operatorname{sel}}(T)} \text { (Select) }
\end{aligned}
$$

We omit the runtime typing rules. By setting $[\Sigma \Sigma]$ as the compositional mapping such that $\llbracket r: \overline{\operatorname{sel}}(T) \rrbracket$ is given as $r: S_{r} \cdot \bar{r}: \overline{S_{r}}$ when $S_{r}=\mu \mathrm{X} . ?(T)$; X, and otherwise identity, as well as extending the notion of error to the internal typecase of the select operation, we obtain, writing $\mathrm{ESP}^{+}$for the extension of ESP with selectors:

\section{Proposition 4.4 (Soundness of Selector Typing Rules).}

1. (Type Preservation) $\Gamma \vdash P \triangleright \Sigma$ in $\mathrm{ESP}^{+}$if and only if $\Gamma \vdash[[P] \triangleright \llbracket \Sigma \rrbracket$.

2. (Soundness) $P \equiv P^{\prime}$ implies $\llbracket P \rrbracket \equiv\left[\left[P^{\prime}\right]\right.$; and $P \longrightarrow P^{\prime}$ implies $\left[[P] \longrightarrow{ }^{*} \llbracket P^{\prime}\right]$.

3. (Safety) A typable process in $\mathrm{ESP}^{+}$never reduces to an error.

$(1,2)$ are straightforward. (3) is a corollary from $(1,2)$ and Theorems 4.2 and 4.3. This example demonstrates how the fine-grained typing rules of ESP can suggest and justify sound typing rules for high-level event handling constructs through ESP encodings.

The typing rules for the switch-receive construct from Example 3.2 can also be derived and justified by its encoding; the details are available from [25].

Event progress. The behaviour of the $\mathrm{ESP}^{+}$selector implicitly features session delegation (session channel passing) as get and put operations on channel collections. The presence of delegation generally makes it impossible to guarantee progress in session typed processes without specialised techniques [4]. ${ }^{5}$ However, we observe that a selector does not use delegation in an arbitrary way; indeed, one of the key characteristics of event-driven programs is their non-blocking nature. In the following, we show that an $\mathrm{ESP}^{+}$-process driven by a selector does satisfy a strong form of progress.

Definition 4.5 (Selector Usage). We say a closed $\mathrm{ESP}^{+}$-process $\Gamma \vdash P \triangleright \Sigma$ uses selectors well if each select action at $r$ is preceded by a register action at $r$, and each register action at $r$ is followed by a select action at $r$, both up to the unfolding of recursion; moreover each select $(r)\left\{\left(x_{i}: S_{i}\right): P_{i}\right\}_{i \in I}$ in $P$ satisfies: (1) (consumption) each $S_{i}$ starts from a branching or linear input and $P_{i}$ starts from the corresponding action on $x_{i}$; and (2) (exhaustiveness) in each $P_{i}$, no input or branching actions occur other than (1).

\footnotetext{
${ }^{5}$ One approach is to impose an order on the channels stored via session channel passing; however, the standard ordering techniques cannot be applied to event selectors (see $\S 6$ ), so progress is difficult to establish in this way.
} 
By the above conditions, all expected events will be handled on every registered channel: as far as the environment generates the events (i.e. produces the messages), the selector will proceed to process them one by one.

We now introduce two conditions from [15]. A typable ESP ${ }^{+}$-process $P$ is simple if the session typings in the premise and conclusion of each prefix rule from Figure 5 in $P$ 's typing derivation are restricted to at most a singleton; and $\Sigma=\emptyset$ in (Selector, Reg,Sel). ${ }^{6}$ We also say $P$ is well-linked if $P \longrightarrow{ }^{*} Q$ implies whenever $Q$ has an active prefix whose subject is a (free or bound) shared name, then the dual active prefix always occurs in $Q$.

We use the refined reduction $\searrow$, defined as $\longrightarrow_{s}^{*} \longrightarrow{ }_{n s} \longrightarrow{ }_{s}^{*}$ where $\longrightarrow_{s}$ is the reduction induced by the last of the selector reduction rules in $\S 3.3$; and $\longrightarrow_{n s}=\longrightarrow \backslash \longrightarrow_{s}$, that is $\longrightarrow_{n s}$ is the whole reduction minus $\longrightarrow_{s}$. We state the event progress theorem in terms of $\searrow$ because $\longrightarrow s$ can still occur in a deadlocked configuration: it does not constitute a "progress step".

Theorem 4.6 (Event Progress). We say an $\mathrm{ESP}^{+}$-process $P$ is eventful if $\Gamma \vdash P \triangleright \emptyset$, it is simple and well-linked, and it uses selectors well in the sense of Definition 4.5. Then we have: (1) if $P$ is eventful and $P \longrightarrow Q$ then $Q$ is eventful; and (2) if $P$ is eventful then either $P \equiv \mathbf{0}$ or $P \searrow Q$ for some $Q$.

This result strictly extends progress for session types to support implicit, well-disciplined usage of session delegation. In addition, all session actions registered with a selector will indeed be processed in a non-blocking fashion, formally justifying the implicit assumptions and expected behaviour of the standard event-driven programming patterns found in practice.

\section{Eventful SJ: Implementation and Evaluation}

This section firstly discusses the design and implementation of Eventful SJ (ESJ). We then report our experience of programming a substantial application in ESJ, a sessiontyped SMTP server, and discuss benchmark results.

\subsection{Event Primitives and ESJ Compilation}

The theoretical inquiries in $\S 3$ and $\S 4$ give a firm formal basis for, and insight on, potential primitives for event-based session programming. The current design of ESJ is based on the selector construct, message arrival predicate and the session typecase. Our selector enhances its untyped counterpart as found in Java NIO and Unix with session-typed operations. In $\S 3$, we have seen that such a selector is encodable using the message arrival predicate through polling: however, polling is inefficient from the performance viewpoint, favouring the introduction of this construct as a primitive.

ESJ is implemented using Polyglot [24], and currently comprises approximately 30 KLOC of Java. First, the compiler statically type checks and transforms ESJ-programs

\footnotetext{
6 This condition precludes the explicit use of delegation and the interleaving of sessions, but can be relaxed to support nested sessions as in [4].
} 


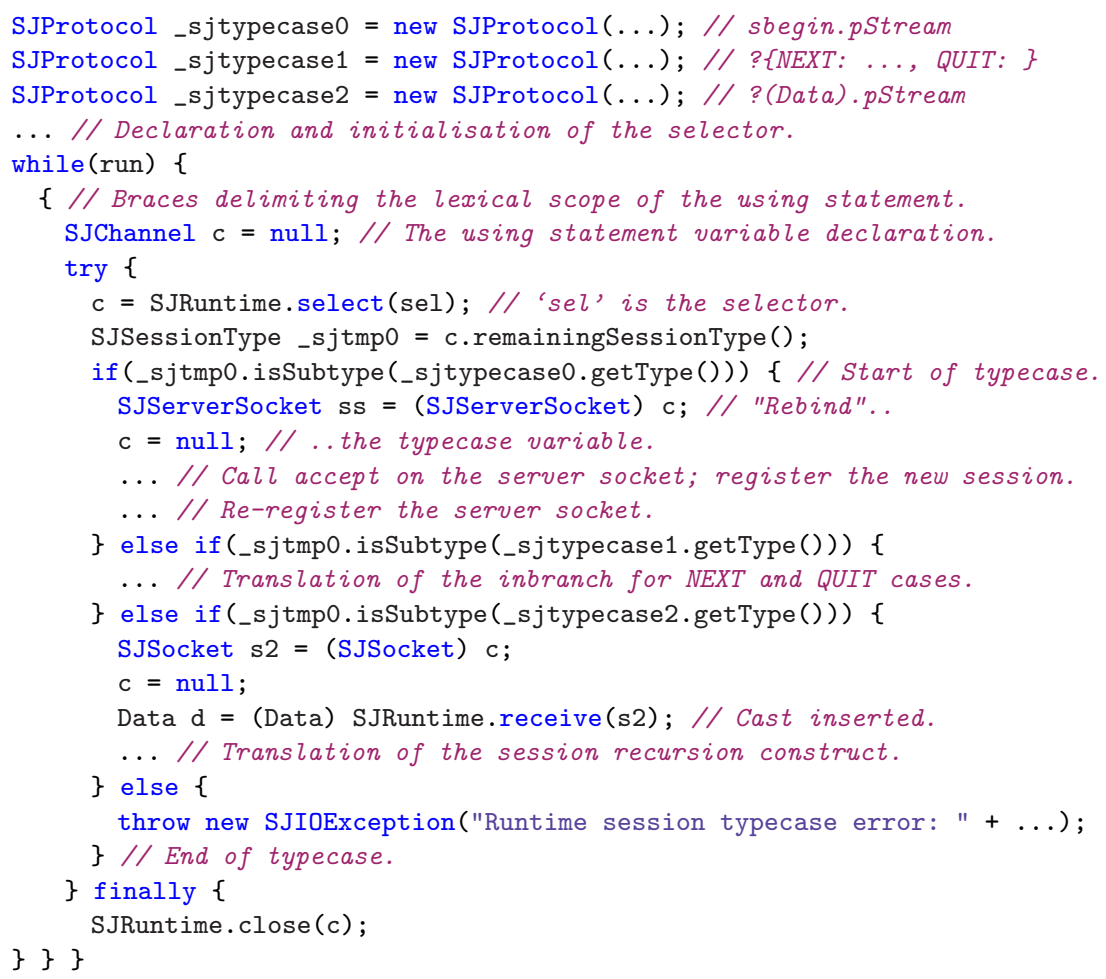

Fig. 6. Compilation of the ESJ "event streams" example from Figure 2 to Java (extract).

into standard Java. The transformation serializes and embeds session type information into the generated classes, and translates the SJ session constructs into transportindependent SJ Runtime (SJR) operations. Then at runtime, the SJR (implemented as a Java library) is used to perform the abstract session operations as actions on a "concrete" transport connection, such as TCP or shared memory: the transport is negotiated at session initiation according to system and user parameters. In this way, the SJ framework supports the execution of SJ programs on any compliant JVM while decoupling type-safe session abstraction from specific modes of transport.

The new ESJ extensions replace the previous compiler with a generalised design based on session set typing (singleton set types subsume the previous non-set types). The use of SJSelector operations and typecase are checked as part of static session type checking following $\S 4.2$ to preserve the safety properties presented in $\S 4.3,4.4$. Here, we illustrate the compiler translation of high-level session constructs into SJR operations using the ESJ "event streams" example from $\S 2$, focusing on the treatment of typecase and SJSelector. Figure 6 extracts from the standard Java code generated by the ESJ compiler for the main event loop. As in C\#, using statements are translated into try-finally statements with appropriate resource cleanup in the finally block, and the lexical scope of using variable declarations is controlled by an outer pair of 
block braces. Basic session operations like select (Line 9) and receive (Line 21) are directly translated into SJR operations, passing the target references (respectively the SJSelector sel and the SJSocket s2) as arguments; similarly for send and arrived.

We now explain the translation of the typecase. First, the session types guarding each case are embedded into the parent class as serialized SJProtocol objects. We then insert a remainingSessionType call to the typecase target $c$ (Line 10) to determine the runtime session type of the SJChannel when the typecase is performed. The structure of the typecase is translated into an if-else statement. Following the formal semantics of typecase ( $(3.2)$, the if-cases find the first typecase-case, according to their original syntactic order, where the current session type of $\mathrm{c}$ is a subtype of the specified type. The "rebinding" of the typecase variable in each case is achieved by inserting an appropriate cast: to SJServerSocket in the first case, and to SJSocket in the second, as directed by their corresponding session types. Note that the rebinding also sets $\mathrm{c}$ to null. Although session types are embedded in their serialized forms, the first call to getType on an SJProtocol decodes the type and caches the value for future use. The final else case serves only as a security measure, since session typing guarantees that at least one of the main cases will match.

\subsection{Eventful SJ Runtime}

SJ Runtime structure and the ESJ extensions. The SJ Runtime (SJR) provides an abstract platform for executing the same high-level, typed session programs over different transports. As described above, the SJ compiler transforms SJ classes into standard java binaries, translating user-level session constructs into SJR operations. The SJR operations target specific Interaction Service (IS) components, which form the upper layer of the SJR, encapsulating services such as session initiation, message serialization, branch synchronisation and session delegation [25]. The IS components are implemented in terms of actions on the Abstract Transport Interface (ATI), which specifies the communication actions of an idealised session transport: bi-directional, order-preserving and reliable delivery of byte segments. The ATI is in turn implemented by Transport components, encapsulating the concrete communication mechanisms of specific transports.

The new ESJ Runtime features several SJR extensions. To support the above translation of typecase (and following the formal semantics), we create an IS service for runtime session type monitoring that tracks the progress of session execution (i.e. the active type, §3.1). The following focuses on the ESJ extensions related to the SJSelector facilities. The challenge is to integrate the infrastructure for asynchronous event handling underlying the SJSelector API in a way that (1) fits the transport-independent SJ framework, and (2) gives the performance expected from event-based systems.

Runtime support for session event selectors. Figure 7 depicts the main IS and Transport components involved in the execution of an event-driven SJ program implemented using the SJSelector API. The key elements of the ESJ extensions are the event-driven ATI extensions, the asynchronous message deserializers, and the abstract selector services.

Event-driven ATI. The ESJ ATI extensions export a TransportSelector interface for detecting asynchronous communication events at the transport level. The implementa- 


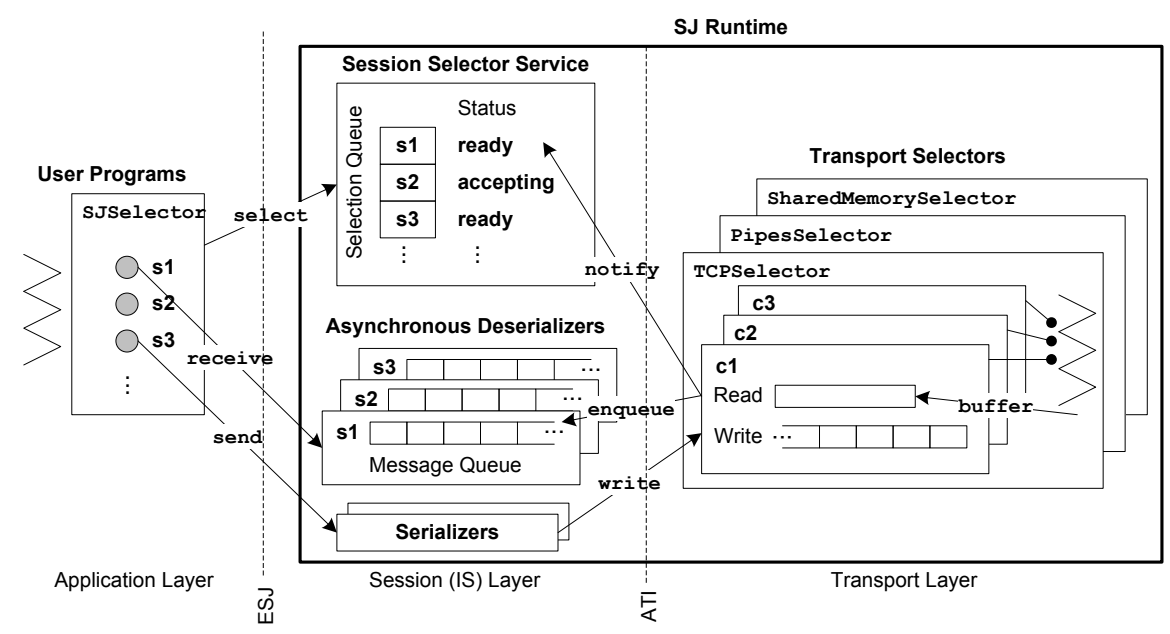

Fig. 7. The main ESJ Runtime components for executing event-driven session programs.

tion of each transport-specific selector is provided by the Transport component that implements the ATI, e.g. the TCPSelector provided by the TCP component uses Java NIO. On initialisation, the SJR creates and maintains one instance of the TransportSelector for each ESJ-compatible transport available. As Figure 7 shows, the ESJ ATI decouples the abstract session selector service (explained below) from the various transports over which the registered sessions are being conducted.

Asynchronous deserializers. Sessions initialised in asynchronous I/O mode (i.e. for event-driven execution via an SJSelector) specify an appropriate asynchronous deserializer component, which encapsulates the routines for converting transport-level binary data into application-level messages. When a read event occurs at the transport-level, the TransportSelector upcalls the deserializer to determine whether enough data for a complete application-level message has been received. If so, the message is enqueued, ready for user consumption, and any remaining data is re-buffered. Otherwise, the data is similarly re-buffered for use on the next read event.

Session selector services. Each SJSelector is supported by an instance of an IS-level SJSelectorService. This service maintains a record of the asynchronous sessions registered with the parent SJSelector, and in turn registers the ATI connection underlying each session with the appropriate TransportSelector. The SJSelectorService is notified by the TransportSelector when an application-level message has been deserialized and enqueued; the SJSelectorService can then report this event when select is called from the application-level.

Sending a message on an asynchronous session uses the serializer to directly enqueue the binary data for writing at the transport-level (i.e. in the local o-buffer); the send operation then immediately returns. Testing session message arrival in the upcalls from the TransportSelector, as opposed to e.g. polling from the IS-layer, is important for fair and efficient event detection in multi-transport contexts. 


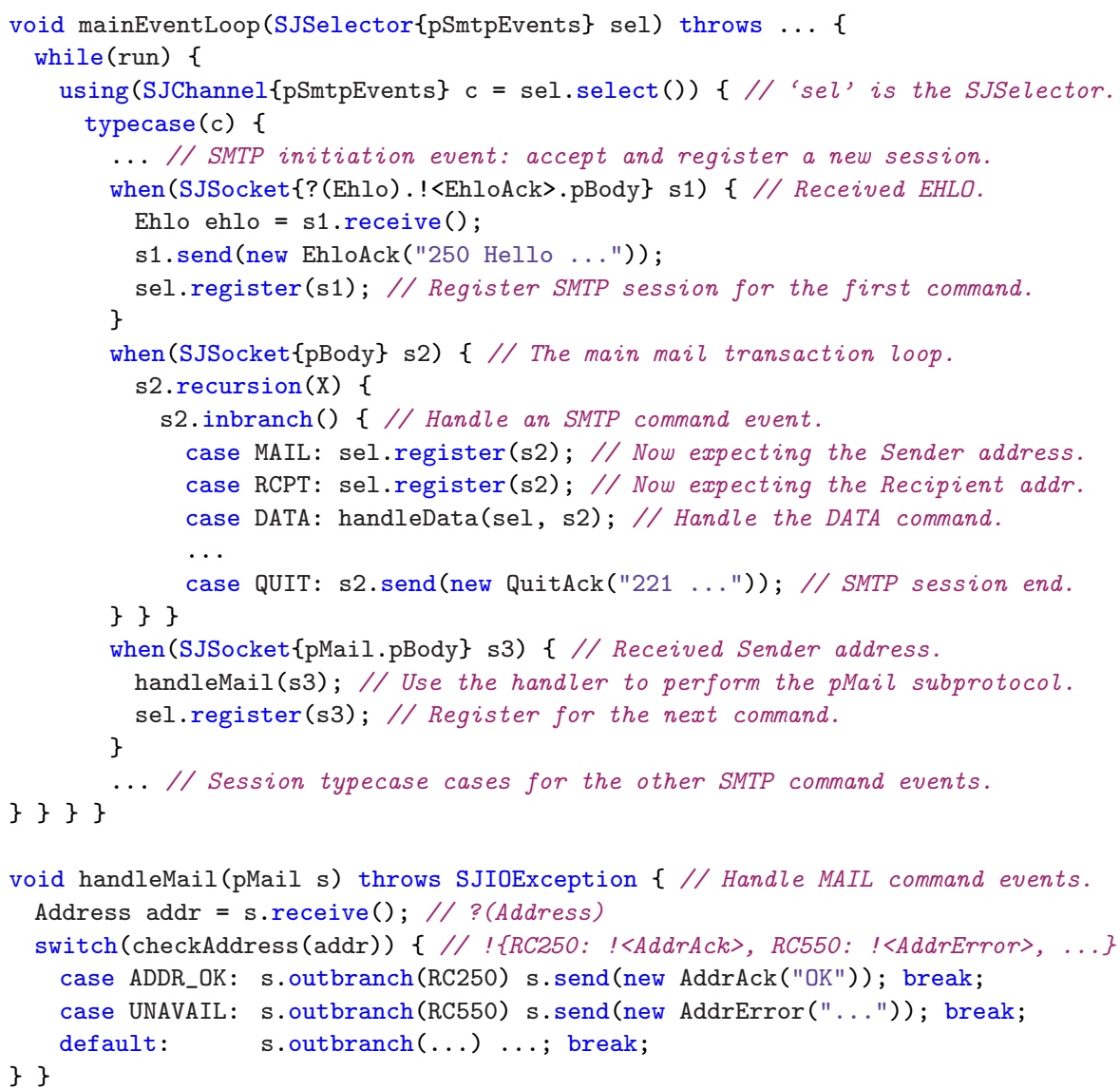

Fig. 9. The main event loop and the MAIL event handler from the ESJ SMTP server.

we pass the session to the omitted handleData method (which sends an RC354 reply before similarly re-registering the session to await the message data). The QUIT branch case sends an acknowledgement, but does not re-register the session since it has now been completed. The final listed when case handles the arrival of the Address message for the MAIL branch by passing the session to the handleMail method, which receives the Address and returns one of the specified reply codes as appropriate. The pMail session type prefix of the s3 argument at the point of the handleMail call corresponds to the session type of the s parameter of the method: this prefix is consumed by the method call and the remaining type of $s 3$ when it is re-registered with sel is again pBody.

Taking advantage of SJ framework modularity, we implement the ESJ SMTP server to be fully interoperable with non-SJ SMTP clients while retaining session type safety. Firstly, we provide application-specific serialization components: we read and write UTF-8, formatted according to the SMTP protocol, e.g. messages are terminated by 
'CRLF'. Each message class, including those for branch labels, provides its own deserialization routine. The SJR uses the current type of each session (tracked by the runtime session monitor) to determine the expected message type(s) and apply the appropriate routine. Note that the operational semantics of enacting a session recursion does not involve any underlying communications (see [Instance] in Figure 4), and hence does not affect the non-SJ peer. Finally, the normal SJ peer compatibility check at session initiation is not possible with non-SJ peers: full communication safety is instead guaranteed through dynamic typing of non-SJ client actions by the session monitoring service.

ESJ programming experience. Our experience of implementing the SMTP server reinforces the following attributes of event-driven session programming.

1. Session types promote clear and safe implementation of complex, real-world protocols featuring branching and recursion. The ability to decompose protocols into subprotocols (e.g. pBody, pMail) and explicit specification of system events (e.g. pSmtpEvents) greatly facilitates the structuring of asynchronous event-driven code. Session types ensure precise identification of event types when handling multiple, concurrent sessions at different stages of execution.

2. Our implementation is guaranteed to conform to the session type specification of SMTP through static typing, and all events will be handled correctly until session completion. Together with session monitoring to verify client conformance (for non-SJ clients), runtime communication safety is guaranteed; we have tested our server against commercial clients such as Microsoft Outlook and Apple Mail.

3. The programmer controls the level of detail at which the protocol is represented by session types. Due to space considerations, the presented SMTP specification is relatively basic: it is fully possible to capture finer-grained details of the SMTP protocol, such as the strict order for MAIL, RCPT and DATA commands, for verification by static type checking. Session set type subtyping also offers a natural mechanism for refining event-driven implementations to support additional event types.

4. The ESJ implementation is inherently cross-transport: the IS services for session events and custom message serialization run above the ATI, and are hence re-usable over different transports, e.g. TCP, TLS, transports for LAN messaging, etc.

We have re-used the above components to implement a type-safe SJ STMP client that is similarly interoperable with existing SMTP servers [25]. Language facilities for the formal declaration and static verification of protocols in communications-based programs will have significant impact on engineering application-level protocols. Richer protocols can be specified with more precision and less effort, supported by automatic static type checking that ensures compatibility between protocol implementations.

ESJ performance. We evaluate the performance of the event-driven ESJ SMTP server against an equivalent multithreaded SJ implementation. The results show that eventdriven session programs exhibit the same performance characteristics (i.e. better scalability under high concurrent loads) as traditional event-driven programming in comparison to their multithreaded counterparts, while maintaining session type safety.

In this macro benchmark, we measure SMTP server throughput in terms of the total number of messages handled by the server while engaged in a varied number of concurrent client sessions. The server is hosted on one machine (locked to one core), and the 


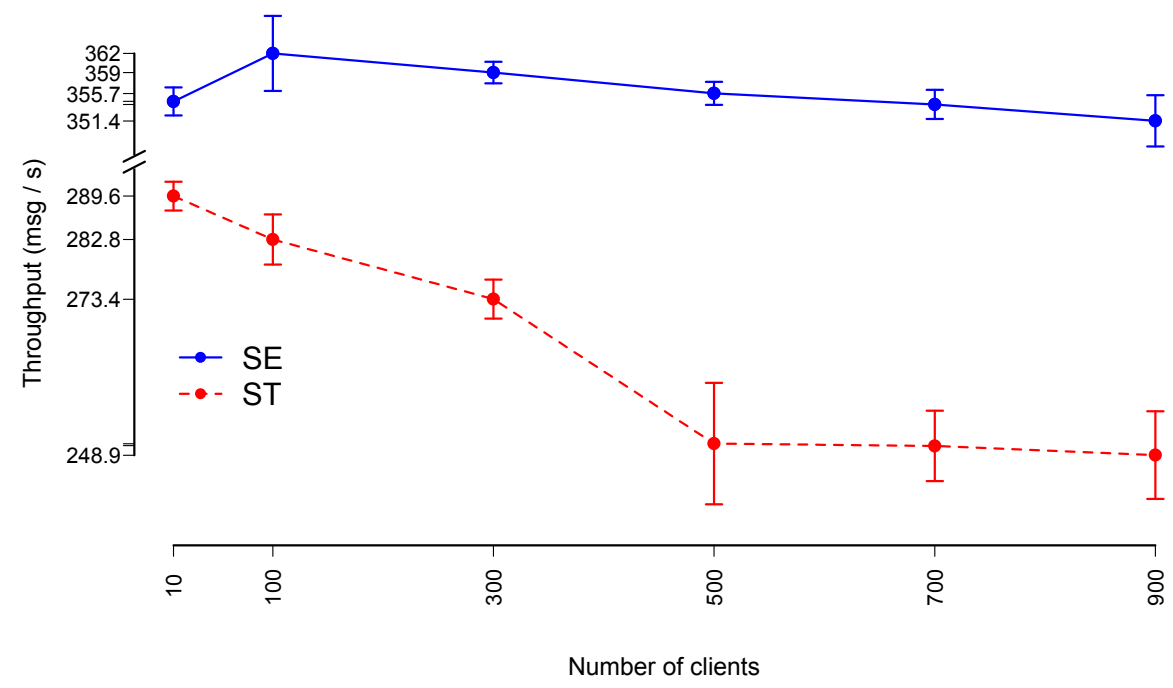

Fig. 10. Mean throughput and throughput standard deviation of multithreaded (ST) and eventdriven (SE) SJ SMTP servers under increasing client loads.

remote clients are set to run continuously repeating mail transaction loops, submitting messages of certain sizes. The benchmark is conducted in a controlled cluster environment: each node is a Sun Fire $\mathrm{x} 4100$ with two 64-bit Opteron 275 processors at $2 \mathrm{GHz}$ and 8 GB of RAM, running 64-bit Mandrakelinux 10.2 (kernel 2.6.11) and connected via gigabit Ethernet. Latency between each node is measured to be $0.5 \mathrm{~ms}$ on average (ping 64 bytes). The benchmark applications are compiled and executed using the Sun Java SE compiler and Runtime versions 1.6.0.

After server performance in the running benchmark configuration has stabilised, the throughput of the server is measured across a series of 50 consecutive 15 second windows; this process is repeated 10 times for each parameter combination, for each the two server implementations. Figure 10 gives the mean throughput and the throughput standard deviation (denoted by the vertical bars) for 10, 100, 300, 500, 700 and 900 concurrent clients and message size 1 KB: the multithreaded SJ server (ST) is the red line, and the event-driven ESJ server (SE) is the blue line. As expected, the results show that the performance of the multithreaded server degrades as the number of clients increases, i.e. mean throughput decreases while throughput variance increases, whereas the throughput of the event-driven SJ server is consistently higher and more stable across all client loads.

The full source code and raw results of this benchmark can be obtained from the SJ homepage [25]. The SJ homepage also presents further micro and macro ESJ benchmark results, including comparisons with multithreaded and event-driven programs implemented in standard Java: the results show that ESJ performs competitively against "untyped event-programming" using NIO. Earlier benchmark results comparing multithreaded SJ against standard TCP sockets and RMI were presented in [16]. 


\section{Related and Future Work}

Language facilities for event-driven programming. Traditional event-driven programming is acknowledged to attain performance and scalability at the cost of complex control flow and manual stack management [2,29]. Consequently, several works have sought to facilitate event-driven programming by adding language features that raise the level of abstraction and/or aid code verification. Tame [20] introduces language features, similar to the synchronisation mechanisms of futures, that allow control flow to be returned from a blocked $\mathrm{C}++$ function to the caller. EventJava [8] integrates advanced event correlation techniques with $\mathrm{O}-\mathrm{O}$ programming, providing high-level syntax for expressing complex patterns of predicated events and a modular framework for implementing alternative semantics for event matching. These works do not offer a characterisation of communication events as enabled by structured sessions nor the associated static safety guarantees of session types. A session type describes not only the pending event type, but also delineates the interaction flow in which the event has occurred, providing strong type-based guarantees such as event-handling safety and progress.

Other recent works have sought to combine elements from both thread and eventbased programming interfaces over event-driven runtimes. A hybrid threads-events system has been embedded into Haskell [22] where both multithreaded and event-driven components are implemented at the application level. The Scala actors library [12] offers both thread-blocking receive operations and actor-based event handlers, decoupled from threads as closures, that "piggy-back" event handling on the source thread that triggers the event. The Capriccio system [30] uses compiler transformations of user-level thread code, replacing blocking I/O with non-blocking equivalents, coupled with efficient runtime stack management to minimise thread overheads. Although these works offer improved runtime support for user-level threads, event-driven programming remains a fundamental paradigm in terms of system design and scalable performance in reactive and high-concurrency communications-based applications such as Web servers $[19,31]$. Unlike these works, the present paper aims not to circumvent, but rather to facilitate event-driven programming through a structured and type-safe programming methodology developed from a formal basis of session types.

Session-typed programming languages and formalisms. Sing\# [9] is a systems-level language with session types for inter-component interaction that features join constructs for handling the arrival of various message patterns. Join patterns are readily encoded into ESP as conjunctions of message arrival predicates as shown in $\S 3.3$. A recent work [11] has proposed a fine-grained integration of object-oriented and session-typed programming: one of the advantages is the ability to store session endpoints as object fields. Their work does not treat event-driven programming, progress or implementation with session endpoint passing (delegation). Session typecase provides an alternative mechanism for supporting sessions as object fields without requiring an additional typing layer (for controlling the order of method calls) as in [11]. A few process typing systems that guarantee advanced progress properties have recently been studied in the context of Web services [4-6]. The present paper is the first to model primitives for type-safe, asynchronous detection of session message arrival and dynamic inspection of session types at runtime. Combining these features enables the assurance of a new progress 
property for asynchronous event handling (event progress, Theorem 4.6), which we apply in practice in our extension of Java for event-driven communications programming. The session channels stored in an ESP selector do not observe a simple partial ordering because they are re-enqeued (i.e. re-registered) depending on asynchronous message arrival and the type of the session; this complex causality, formed through session delegations, is not typable in the previous work.

Future work. The theoretical basis and practical framework for event-driven session programming presented in this paper opens up several directions for further research. One is the extension of both the theory and implementation to support event handling for multiparty sessions [15]: combining the advanced event correlation facilities of [8] with multiparty session types would enable type-safe multicasting and correlation of multiple, heterogeneous sessions. Our mechanisms for the type-safe storage and retrieval of sessions may also serve as a basis for other high-level facilities such as session hibernation and process migration [27]. We are currently designing SJ Runtime extensions for session event selector thread pools, where we assign particular event types (i.e. session types) to specific threads. Session types can be exploited in this role for performance gains (e.g. thread locality for event handling routines) and to facilitate system profiling and load balancing. We are also continuing the practical evaluation of ESJ through the implementation of event-driven applications and further benchmarks [25]. These investigations will assist future developments in the design of safe, high-level language support for managing complex, asynchronous control flow in communicationcentred programming.

Acknowledgements. We thank the ECOOP referees for their comments. This work is partially supported by EPSRC EP/F003757, EP/F002114, EP/G015635 and EP/G015481.

\section{References}

1. M. Abadi, L. Cardelli, B. C. Pierce, and G. D. Plotkin. Dynamic typing in a statically typed language. TOPLAS, 13(2):237-268, 1991.

2. A. Adya, J. Howell, M. Theimer, W. J. Bolosky, and J. R. Douceur. Cooperative task management without manual stack management or, event-driven programming is not the opposite of threaded programming. In USENIX ATC 2002, pages 289-302. USENIX Association, 2002.

3. N. Benton, L. Cardelli, and C. Fournet. Modern concurrency abstractions for C\#. TOPLAS, 26(5):769-804, 2004.

4. L. Bettini et al. Global progress in dynamically interleaved multiparty sessions. In CONCUR 2008, volume 5201 of LNCS, pages 418-433. Springer, 2008.

5. L. Caires and H. T. Vieira. Conversation types. In ESOP 2009, volume 5502 of LNCS, pages 285-300. Springer, 2009.

6. G. Castagna and L. Padovani. Contracts for mobile processes. In CONCUR 2009, volume 5710 of $L N C S$, pages 211-228. Springer, 2009.

7. M. Dezani-Ciancaglini, D. Mostrous, N. Yoshida, and S. Drossopoulou. Session types for object-oriented languages. In ECOOP 2006, volume 4067 of LNCS, pages 328-352. Springer, 2006. 
8. P. Eugster and K. R. Jayaram. Eventjava: An extension of java for event correlation. In ECOOP, volume 5653 of $L N C S$, pages 570-594. Springer, 2009.

9. M. Fähndrich, M. Aiken, C. Hawblitzel, O. Hodson, G. Hunt, J. R. Larus, and S. Levi. Language support for fast and reliable message-based communication in singularity os. In $\mathrm{Eu}$ roSys 2006, ACM SIGOPS, pages 177-190. ACM, 2006.

10. C. Fournet, C. Laneve, L. Maranget, and D. Rémy. Inheritance in the join calculus. JLAP, 57(1-2):23-69, 2003.

11. S. J. Gay, V. T. Vasconcelos, A. Ravara, N. Gesbert, and A. Z. Caldeira. Modular session types for distributed object-oriented programming. In POPL 2010, volume 45, pages 299312. ACM, 2010.

12. P. Haller and M. Odersky. Scala actors: Unifying thread-based and event-based programming. Theoretical Computer Science, 410(2-3):202-220, 2009.

13. K. Honda and M. Tokoro. An object calculus for asynchronous communication. In ECOOP 1991, volume 512 of LNCS, pages 133-147. Springer-Verlag, 1991.

14. K. Honda, V. T. Vasconcelos, and M. Kubo. Language primitives and type disciplines for structured communication-based programming. In ESOP 1998, volume 1381 of LNCS, pages 122-138. Springer, 1998.

15. K. Honda, N. Yoshida, and M. Carbone. Multiparty asynchronous session types. In POPL 2008, pages 273-284. ACM, 2008.

16. R. Hu, N. Yoshida, and K. Honda. Session-based distributed programming in java. In ECOOP 2008, volume 5142 of LNCS, pages 516-541. Springer, 2008.

17. Java New I/O APIs. http://java.sun.com/j2se/1.4.2/docs/guide/nio/.

18. D. Kouzapas. A session type discipline for event driven programming models. Master's thesis, Imperial College London, 2009. http://www.doc.ic.ac.uk/teaching/distinguishedprojects/2010/d.kouzapas.pdf.

19. M. Krohn. Building secure high-performance web services with okws. In USENIX ATC 2004, pages 185-198. USENIX Association, 2004.

20. M. Krohn, E. Kohler, and M. F. Kaashoek. Events can make sense. In USENIX ATC 2007, pages 1-14. USENIX Association, 2007.

21. H. C. Lauer and R. M. Needham. On the duality of operating system structures. SIGOPS Operating Systems Review, 13(2):3-19, 1979.

22. P. Li and S. Zdancewic. Combining events and threads for scalable network services implementation and evaluation of monadic, application-level concurrency primitives. SIGPLAN Not., 42(6):189-199, 2007.

23. D. Mostrous and N. Yoshida. Session-based communication optimisation for higher-order mobile processes. In TLCA 2009, volume 5608 of LNCS, pages 203-218. Springer, 2009.

24. Polyglot homepage. http://www.cs.cornell.edu/Projects/polyglot/.

25. SJ homepage. http://www.doc.ic.ac.uk/ rhu/sessionj.html.

26. The simple mail transfer protocol. http://tools.ietf .org/html/rfc5321.

27. A. C. Snoeren and H. Balakrishnan. An end-to-end approach to host mobility. In MOBICOM 2000, pages 155-166. ACM, 2000.

28. K. Takeuchi, K. Honda, and M. Kubo. An interaction-based language and its typing system. In PARLE 1994, volume 817 of LNCS, pages 398-413. Springer-Verlag, 1994.

29. R. von Behren, J. Condit, and E. Brewer. Why events are a bad idea (for high-concurrency servers). In HOTOS 2003, pages 4-4. USENIX Association, 2003.

30. R. von Behren, J. Condit, F. Zhou, G. C. Necula, and E. Brewer. Capriccio: scalable threads for internet services. In SOSP 2003, pages 268-281. ACM, 2003.

31. M. Welsh, D. E. Culler, and E. A. Brewer. Seda: An architecture for well-conditioned, scalable internet services. In SOSP 2001, pages 230-243. ACM, 2001. 